\title{
DOENÇAS CARDIOVASCULARES: FATORES DE RISCO EM ADOLESCENTES
}

\author{
Bartira Bezerra de Brito ${ }^{1}$, Jéssica Denise Vieira Leal ${ }^{2}$, Laura Maria Feitosa Formiga ${ }^{3}$, Karoline de Macêdo \\ Gonçalves Frota ${ }^{4}$, Ana Roberta Vilarouca da Silva ${ }^{5}$, Luisa Helena de Oliveira Lima ${ }^{5}$
}

\begin{abstract}
RESUMO: O objetivo do estudo foi investigar os fatores de risco para doenças cardiovasculares em adolescentes do município de Picos-Piauí. Estudo descritivo e transversal realizado com 151 adolescentes. A coleta de dados ocorreu no período de agosto a outubro de 2013 nas escolas particulares. Os resultados evidenciaram que há presença de alterações na pressão arterial nos indivíduos do sexo masculino e feminino, 8,6\% e 14,8\%, respectivamente, com os valores entre os percentis 90 e 95, classificando como limítrofe. A maioria da amostra apresentou excesso de peso. O perfil lipídico estava alterado em boa parte da população estudada. Com base nesses achados, torna-se necessário a formulação de estratégias de prevenção para os adolescentes visando à promoção de estilos de vida saudáveis como dietas com baixa densidade energética e incremento na atividade física.
\end{abstract}

DESCRITORES: Doenças cardiovasculares; Fatores de risco; Adolescentes; Prevalência.

\section{CARDIOVASCULAR DISEASES: RISK FACTORS IN ADOLESCENTS}

\begin{abstract}
The objective of this study was to investigate risk factors for cardiovascular diseases in adolescents in the city of Picos, in the state of Piauí, Brazil. A descriptive and cross-sectional study was conducted with 151 adolescents. Data were collected from August to October of 2013 in private schools. The results showed that there are variations in blood pressure of $8.6 \%$ and $14.8 \%$ in men and women, respectively, with values from $90 \%$ to $95 \%$, classifying as bordering high. The majority of the sample presented overweight. The lipid profile of the majority of the population studied was altered. Based on these facts, the development of prevention strategies for adolescents is necessary, aiming to promote healthy lifestyles, such as low-calorie diets and increase of physical activity.
\end{abstract}

DESCRIPTORS: Cardiovascular diseases; Risk factors; Adolescents; Prevalence.

\section{ENFERMEDADES CARDIOVASCULARES: FACTORES DE RIESGO EN ADOLESCENTES}

RESUMEN: El objetivo de este estudio fue investigar los factores de riesgo a las enfermedades cardiovasculares en adolescentes del municipio de Picos-Piauí. Estudio descriptivo y transversal, realizado con 151 adolescentes. Datos recolectados durante el período de agosto a octubre de 2013 en las escuelas particulares. Los resultados evidenciaron que existe presencia de alteraciones en la presión arterial de los individuos de sexo masculino y femenino, $8,6 \%$ y $14,8 \%$ respectivamente, con los valores entre los percentiles 90 y 95 clasificando como limítrofes. La mayoría de la muestra presentó exceso de peso. El perfil lipídico estaba alterado en buena parte de la población estudiada. En base a tales hallazgos, resulta necesaria la formulación de estrategias de prevención para los adolescentes, apuntando a la promoción de estilos de vida saludables, incluyendo dietas de baja densidad energética e incremento de la actividad física.

DESCRIPTORES: Enfermedades Cardiovasculares; Factores de Riesgo; Adolescente; Prevalencia.

${ }^{1}$ Enfermeira. Enfermeira da equipe de saúde da família do centro integrado de saúde. Secretaria de Saúde do Município de Fronteiras. Fronteiras, PI, Brasil

${ }^{2}$ Enfermeira. Mestranda em Ciências e Saúde. Universidade Federal do Piauí. Teresina, PI, Brasil.

${ }^{3}$ Enfermeira. Mestre em Farmacologia Clínica. Docente de Enfermagem da Universidade Federal do Piauí. Picos, PI, Brasil.

${ }^{4}$ Nutricionista. Doutora em Nutrição em Saúde Pública. Docente de Nutrição da Universidade Federal do Piauí. Teresina, PI, Brasil.

${ }^{5}$ Enfermeira. Doutora em Enfermagem. Docente de Enfermagem da Universidade Federal do Piauí. Picos, PI, Brasil.

Autor Correspondente:

Luisa Helena de Oliveira Lima

Universidade Federal do Piauí

R. Zuza Lino, 1239 - 64600-160 - Picos, PI, Brasil

E-mail: luisa17lima@gmail.com
Recebido: $27 / 06 / 2015$

Finalizado: $28 / 04 / 2016$ 


\section{- INTRODUÇÃO}

No último século, o crescimento acelerado do processo de urbanização, ligado à industrialização e ao desenvolvimento tecnológico, acarretou em muitos países modificações no estilo de vida da população como, por exemplo, adoção de hábitos alimentares inadequados e inatividade física. Esse contexto contribuiu para uma alteração nas principais causas de mortalidade e morbidade, com predominância das Doenças Crônicas Não Transmissíveis (DCNTs) em relação às doenças infectocontagiosas.

Essas doenças são um problema de saúde global e uma ameaça à saúde e ao desenvolvimento humano. Sua carga recai especialmente sobre países de baixa e média renda ${ }^{(1)}$.

No Brasil, as DCNTs e as causas externas foram as principais causas de morte em 2009, correspondendo a $85,0 \%$ do total de óbitos. Dentre as mortes por DCNTs, as principais causas são as Doenças Cardiovasculares (DCVs), principalmente as isquêmicas do coração, as hipertensivas e as cerebrovasculares. Segundo o Plano de ações estratégicas para o enfrentamento das doenças crônicas não transmissíveis (DCNT) no Brasil, 31,3\% das pessoas morreram por essas doenças em $2008^{(2,3)}$.

O impacto das DCVs será maior nos países em desenvolvimento, como o Brasil, pois contribuem mais sobre o seu ônus, em relação aos países desenvolvidos. Há previsão de que nos países desenvolvidos o risco de morte deve aumentar 20\% até 2020, e naqueles em desenvolvimento deverá dobrar. Segundo dados da Universidade de Colúmbia e do Banco Mundial, se forem mantidas as atuais projeções, o Brasil terá as maiores taxas de novos eventos do mundo nas próximas décadas ${ }^{(4)}$.

As DCVs têm origem multifatorial em que os chamados fatores de risco participam de sua gênese. Fatores de risco são variáveis que predispõem ao aparecimento e ao desenvolvimento das cardiopatias; logo, sua monitoração auxilia na identificação de sinais precursores que, ao serem modificados, podem diminuir ou até mesmo reverter o processo evolutivo das disfunções ${ }^{(5)}$.

Estes fatores de risco podem ser classificados em modificáveis, tais como o tabagismo, colesterol sérico elevado, hipertensão arterial sistêmica, inatividade física, diabetes, obesidade, estresse, uso de anticoncepcional e obesidade abdominal; e não modificáveis sendo estes, hereditariedade, sexo e idade avançada ${ }^{(4)}$.

Tais fatores de risco presentes nos jovens estão relacionados a fatores comportamentais como as alterações nos hábitos de atividade física resultando em aumentos nos índices de inatividade, além das mudanças nos hábitos alimentares, principalmente com o aumento no consumo de lipídeos. Os adolescentes estão expostos aos fatores de risco supracitados, principalmente no que diz respeito a problemas nutricionais, como o excesso de peso e o desenvolvimento de obesidade precoce ${ }^{(6,7)}$.

A adolescência se caracteriza como um período propício para o desenvolvimento de estratégias intervencionistas voltadas ao combate das DCVs, uma vez que há evidências de que estas doenças podem se originar neste período de vida. Além disso, diversos fatores de risco de origem biológica adquiridos na adolescência tendem a persistir até a maioridade, acentuando o risco de morbimortalidade na vida adulta.

Assim sendo, a identificação precoce de fatores de risco cardiovascular é de fundamental importância para que possam ser realizadas ações de promoção da saúde e o monitoramento em adolescentes brasileiros. Os resultados desta pesquisa contribuirão sobremaneira para o maior conhecimento da situação epidemiológica neste grupo etário e para a tomada de decisões tanto na área da saúde como na da educação.

A enfermagem enquanto profissão, também deve se preocupar com a questão do surgimento de fatores de riscos em idades cada vez mais jovens, atuando de forma a prevenir o desenvolvimento dos mesmos, colaborando para uma diminuição da morbimortalidade causada pelas DCVs.

Nesta perspectiva, este estudo investigou os fatores de risco para doenças cardiovasculares em adolescentes de escolas particulares do Município de Picos - Piauí. 
Trata-se de um estudo transversal de cunho descritivo, realizado no período de maio a novembro de 2013, nas escolas de ensino fundamental e médio privadas do município de Picos - PI. Participaram da pesquisa apenas as 8 escolas particulares que aceitaram participar do estudo concedendo autorização institucional. As escolas públicas foram objeto de estudo anterior.

A população foi composta por todos os adolescentes de 10 a 19 anos regularmente matriculados nas escolas particulares da zona urbana. Para o cálculo do tamanho da amostra, utilizou-se a fórmula para estudos transversais com população finita ${ }^{(8)}: \mathrm{n}=(\mathrm{Za} 2 * \mathrm{P} * \mathrm{Q} * \mathrm{~N}) /(\mathrm{Za} 2 * \mathrm{P} * \mathrm{Q})+(\mathrm{N}-1) *$ E2. Onde: $\mathrm{n}$ = tamanho da amostra; $\mathrm{Z} a=$ coeficiente de confiança;

$\mathrm{N}=$ tamanho da população; $\mathrm{E}=$ erro amostral absoluto; $\mathrm{Q}=$ porcentagem complementar (100-P); $\mathrm{P}$ = proporção de ocorrência do fenômeno em estudo.

Foram considerados como parâmetros o coeficiente de confiança de 95\% (1,96), o erro amostral de $3 \%$ e população de 2250 adolescentes ( 10 a 19 anos) $(N=2250)$. A prevalência considerada foi a menor prevalência esperada dentre as variáveis escolhidas para estudo $(7 \%$ para hipertensão arterial $)(P=0,07)$ (9). A partir da aplicação da fórmula encontrou-se um total de 151 participantes.

Os participantes foram proporcionalmente selecionados de acordo com o número de alunos matriculados em cada escola. Como critérios de inclusão foram definidos: adolescente com idade entre 10 e 19 anos, cujo responsável aceite participar da pesquisa e assine o termo de consentimento livre e esclarecido. Os critérios de exclusão foram ser filho de pais adotivos, o que dificulta a coleta dos dados a respeitos de antecedentes familiares para DCV. A amostra foi selecionada de forma aleatória de todos os adolescentes que preencheram os critérios de elegibilidade nas instituições escolares.

Os dados foram coletados de agosto a outubro de 2013, por alunas do Curso de Enfermagem e Nutrição devidamente treinadas com técnicas padronizadas. Foram utilizados um formulário (contendo informações sobre identificação dos adolescentes, antropometria medidas hemodinâmicas, hábitos alimentares e atividade física) e um questionário (contendo informações sobre dados de saúde dos pais biológicos, hábitos alimentares) adaptados de outro estudo ${ }^{(10)}$.

A pressão arterial (PA) foi verificada pelo método auscultatório clássico, utilizando um esfigmomanômetro aneroide postado ao nível do coração e um estetoscópio localizado acima da artéria braquial. A pressão arterial sistólica foi definida como o som de Korotkoff fase 1(K1) e a diastólica como o som de Korotkoff fase 5 (K5) ou o desaparecimento de sons ${ }^{(6)}$.

A verificação seguiu os procedimentos recomendados nas VI Diretrizes Brasileiras de Hipertensão (2010). Foram utilizados manguitos de tamanho apropriado à circunferência dos braços das crianças e adolescentes. Para a classificação da pressão foram consideradas as curvas para determinação do percentil da estatura do adolescente de acordo com a idade e o sexo, segundo o National High Blood Pressure Education Program dos Estados Unidos (NHBPEP, 2004) e a tabela de percentil da pressão arterial referenciada pela V Diretrizes de Hipertensão Arterial (2006) ${ }^{(11-13)}$.

Após um jejum noturno de 12 horas, uma coleta de sangue venoso foi realizada nas dependências das escolas para posterior análise bioquímica. As amostras eram acondicionadas em tubos a vácuo com gel separador e sem anticoagulante. Após os procedimentos de coleta, o sangue era centrifugado por 10 minutos a $3.000 \mathrm{rpm}$ para separar o soro dos demais componentes, sendo o soro utilizado para as análises. Os triglicerídeos e a glicose sanguínea foram dosados utilizando um kit enzimático colorimétrico processado no aparelho Autohumalyzer A5 (Human-2004).

O exame foi realizado de acordo com as determinações da I Diretriz de Prevenção da Aterosclerose na Infância e na Adolescência. Os valores de referência utilizados foram: Colesterol total: <150 mg/ dL desejável, 150-169 mg/dL limítrofe e > 170 aumentado. LDL-C: $<100 \mathrm{mg} / \mathrm{dL}$ desejável, 100-129 mg/dL limítrofe, > $130 \mathrm{mg} / \mathrm{dL}$ aumentado. HDL-C: > $45 \mathrm{mg} / \mathrm{dL}$ desejável. Triglicerídeos: <100 mg/dL desejável, 100-129 mg/dL limítrofe, > $130 \mathrm{mg} / \mathrm{dL}$ aumentado. Para a glicemia foi adotado: <100 mg/dL normal, 100-125 mg/dL tolerância à glicose diminuída, $>126 \mathrm{mg} / \mathrm{dL}$ diabetes mellitus ${ }^{(14,15)}$. Esses exames foram custeados pelos próprios pesquisadores. 
Os dados foram analisados com base na literatura específica e em tratamento estatístico, e apresentados em tabelas. Primeiramente foi elaborado banco de dados na planilha Microsoft Office Excel 2010 e posteriormente transportados para o software SPSS versão 20.0. Para análise foi utilizada a estatística descritiva.

Para a realização do estudo, seguimos todos os princípios éticos contidos na Resolução 466/12 que rege pesquisas envolvendo seres humanos ${ }^{(16)}$. O projeto foi devidamente aprovado pelo Comitê de Ética e Pesquisa da Universidade Federal do Piauí ( $\mathrm{N}^{\circ}$ do parecer: 352.372). Todos os participantes que aceitaram participar do estudo assinaram o Termo de Consentimento Livre e Esclarecido e o Termo de Assentimento Livre Esclarecido para adolescentes menores de 18 anos.

\section{RESULTADOS}

Os dados coletados foram organizados de acordo com os grupos de respostas, apresentados na forma de tabelas e analisados utilizando a estatística descritiva.

A Tabela 1 mostra um predomínio de adolescentes do sexo feminino $(53,6 \%)$, sendo a maioria de cor parda (44,4\%). A faixa etária que prevaleceu foi de 10 a 14 anos (72,84\%), com idade mediana de 13 anos.

Os dados observados na Tabela 2 mostraram que $64,3 \%$ e 50,6\% dos adolescentes masculinos e femininos, respectivamente, apresentavam excesso de peso (obesidade e sobrepeso).

Os dados mostraram que em ambos os sexos observou-se níveis pressóricos alterados. Considerando a hipertensão estágio 1 e 2, 15,7\% dos meninos e 14,8\% das meninas apresentaram PA elevada, conforme Tabela 3.

Tabela 1 - Distribuição da amostra de acordo com dados de identificação dos adolescentes $n=151$. Picos, PI, Brasil 2013

\begin{tabular}{lccc} 
Variáveis & F & $\%$ & $\begin{array}{c}\text { Mediana } \\
\text { (IQ) }\end{array}$ \\
\hline Sexo & & & \\
\hline Feminino & 81 & 53,6 & \\
\hline Masculino & 70 & 46,4 & \\
\hline Idade (em anos) & & & \\
\hline $10-14$ & 110 & 72,84 & $13(2)$ \\
\hline $15-19$ & 41 & 27,16 & \\
\hline Cor/Grupo Étnico & & & \\
\hline Branco & 51 & 33,8 & \\
\hline Preto & 24 & 15,9 & \\
\hline Pardo & 67 & 44,4 & \\
\hline Indígena & 2 & 1,3 & \\
\hline Não respondeu & 7 & 4,6 & \\
\hline Total & 151 & 100 &
\end{tabular}

Tabela 2 - Distribuição dos adolescentes, por classificação nutricional baseado no IMC n=151. Picos, PI, Brasil 2013

\begin{tabular}{lcccc}
\multirow{2}{*}{ IMC } & \multicolumn{2}{c}{ Meninos } & \multicolumn{2}{c}{ Meninas } \\
\cline { 2 - 5 } & $\mathbf{f}$ & $\mathbf{\%}$ & $\mathbf{F}$ & $\%$ \\
\hline Magreza extrema & 1 & 1,4 & - & - \\
\hline Magreza & 1 & 1,4 & 1 & 1,2 \\
\hline Eutrofia & 23 & 32,9 & 39 & 48,1 \\
\hline Sobrepeso & 26 & 37,1 & 27 & 33,3 \\
\hline Obesidade & 19 & 27,2 & 14 & 17,3 \\
\hline Total & 70 & 100 & 81 & 100
\end{tabular}

Tabela 3 - Distribuição dos adolescentes de acordo com a classificação da Pressão Arterial n=151. Picos, PI, Brasil 2013

\begin{tabular}{lcccc} 
Classificação da PA & \multicolumn{2}{c}{ Meninos } & \multicolumn{2}{c}{ Meninas } \\
\cline { 2 - 5 } & $\mathbf{f}$ & $\mathbf{\%}$ & $\mathbf{F}$ & $\%$ \\
\hline Normal & 46 & 65,7 & 57 & 70,4 \\
\hline Limítrofe & 13 & 18,6 & 12 & 14,8 \\
\hline Hipertensão estágio 1 & 7 & 10 & 8 & 9,9 \\
\hline Hipertensão estágio 2 & 4 & 5,7 & 4 & 4,9 \\
\hline Total & 70 & 100 & 81 & 100
\end{tabular}


Com relação à caracterização do perfil lipídico e glicídico, merecem destaque os percentuais de alteração encontrados, visto que a adolescência é um período hígido, no qual não se espera alterações tão significativas. De acordo com Tabela 4, os marcadores mais elevados foram Colesterol total $(57,1 \%)$, LDL-colesterol (35\%) e HDL-colesterol baixo (32,9\%).

Com relação à caracterização do perfil lipídico e glicídico, merecem destaque os percentuais de alteração encontrados, visto que a adolescência é um período hígido, no qual não se espera alterações tão significativas. De acordo com Tabela 4, os marcadores mais elevados foram Colesterol total $(57,1 \%)$, LDL-colesterol (35\%) e HDL-colesterol baixo (32,9\%).

Conforme mostrado na Tabela 5, em relação à história familiar para doenças cardiovasculares, considerando os pais, 13,9\% tinham hipertensão, 7,3\% diabetes e $2 \%$ cardiopatia. Já entre as mães, 15,9\% tinham hipertensão e 1,3\% cardiopatia. Embora estas prevalências tenham sido baixas, chama-se atenção para o fato de serem pessoas adultas ainda jovens, pois a média de idade das mães foi de 39,6 anos (dado não mostrado na tabela), e 1,3\% já apresentam cardiopatia.

Tabela 4 - Caracterização do perfil lipídico e glicídico da amostra $n=140$. Picos, PI, Brasil 2013

\begin{tabular}{|c|c|c|}
\hline Exames bioquímicos & $\mathbf{F}$ & $\%$ \\
\hline \multicolumn{3}{|l|}{ Triglicerídeos } \\
\hline Desejável & 98 & 70 \\
\hline Limítrofe & 28 & 20 \\
\hline Aumentado & 14 & 10 \\
\hline \multicolumn{3}{|l|}{ Glicemia } \\
\hline Normal & 136 & 97,1 \\
\hline Tolerância à glicose diminuída & 4 & 2,9 \\
\hline \multicolumn{3}{|l|}{ Colesterol total } \\
\hline Desejável & 34 & 24,3 \\
\hline Limítrofe & 26 & 18,6 \\
\hline Aumentado & 80 & 57,1 \\
\hline \multicolumn{3}{|l|}{ LDL-C } \\
\hline Desejável & 49 & 35 \\
\hline Limítrofe & 42 & 30 \\
\hline Aumentado & 49 & 35 \\
\hline \multicolumn{3}{|l|}{ HDL-C } \\
\hline Desejável & 94 & 67,1 \\
\hline Baixo & 46 & 32,9 \\
\hline
\end{tabular}

Tabela 5 - Distribuição da amostra de acordo com a história familiar para doenças cardiovasculares. $\mathrm{n}=151$. Picos, PI, Brasil 2013

\begin{tabular}{lcccc} 
Parentesco & \multicolumn{2}{c}{ Mãe } & \multicolumn{2}{c}{ Pai } \\
\cline { 2 - 5 } & $\mathbf{F}$ & $\mathbf{\%}$ & $\mathbf{F}$ & $\%$ \\
\hline Doenças & & & & \\
\hline Diabetes & - & - & 11 & 7,3 \\
\hline Hipertensão & 24 & 15,9 & 21 & 13,9 \\
\hline Cardiopata & 2 & 1,3 & 3 & 2
\end{tabular}

\section{- DISCUSSÃO}

Foi avaliado um grupo de adolescentes estudantes de escolas privadas, num total de 151, para investigar a presença de fatores de riscos cardiovascular nesses indivíduos, já que existe uma forte correlação entre a existência e a gravidade de uma DCV e a presença de seus fatores nos estágios inicias da vida. Isso aumenta a preocupação em detectar precocemente esses fatores, prevenindo futuras complicações.

Ao analisarmos os resultados, observamos que $72,84 \%$ dos adolescentes tinham idade entre 10 e 14 anos. A amostra era formada principalmente por adolescentes do sexo feminino (53,6\%), fato semelhante foi observado no estudo realizado no município de João Pessoa - PB, onde 54,9\% da amostra eram do sexo feminino e observado também em outros estudos ${ }^{(17-20)}$. A cor autodeclarada mais prevalente foi a parda, com $44,4 \%$, isso se deve ao fato de o Brasil ser um país formado pela mistura de raças. 
Ao caracterizarmos os adolescentes através do índice de massa corporal (IMC), verificou-se uma prevalência de sobrepeso e obesidade de $37,1 \%$ e $27,2 \%$, respectivamente no sexo masculino e $33,3 \%$ e $17,5 \%$ de sobrepeso e obesidade, respectivamente, no sexo feminino.

O número de adolescentes com excesso de peso é significativo, nesse estudo. Diferente de outro estudo, também realizado com escolares, onde os adolescentes estudados apresentaram 16,7\% e $11,1 \%$ de prevalência de sobrepeso e obesidade respectivamente, nos do sexo masculino, e $7,6 \%$ e $5,3 \%$ de prevalência de sobrepeso e obesidade respectivamente, nos do sexo feminino ${ }^{(21)}$.

Relacionando o IMC na infância e o risco de eventos coronarianos na vida adulta, mostra-se que cada unidade aumentada no IMC eleva a probabilidade da ocorrência de eventos coronarianos futuros, e essa associação aumenta com a idade ${ }^{(3)}$.

Neste estudo, foi verificada a presença de alterações na pressão arterial nos indivíduos do sexo masculino e feminino, no qual $18,6 \%$ e $14,8 \%$, respectivamente estavam com os valores entre os percentis 90 e 95, classificando como limítrofe (pré-hipertensão). Além de 15,7\% dos meninos e 14,8\% das meninas apresentarem PA elevada.

Em outro trabalho também realizado na cidade de Picos, com alunos da rede pública de ensino, a porcentagem de adolescentes com PA limítrofe foi de $38,6 \%{ }^{(7)}$.

A hipertensão arterial é relacionada como uma importante doença crônica da população adulta e geriátrica. Mas durante os últimos 30 anos, a prevalência cresce na faixa etária infanto-juvenil no cenário mundial. Uma publicação nacional demonstra prevalência de pressão arterial elevada em crianças e adolescentes, que variam entre $1-12 \%$ e $2-10 \%$, respectivamente ${ }^{(17)}$.

Apesar dos níveis da pressão arterial alterados não determinarem, necessariamente, um diagnóstico de hipertensão arterial sistêmica (HAS), o fato pode apontar para um risco de desenvolver problema cardiovascular na fase adulta. Para criança com esse problema, por exemplo, as chances dela se tornar uma adulta hipertensa são 2,4 vezes maiores em relação às demais ${ }^{(17)}$.

A alteração dos perfis lipídicos e glicídico foi observada em boa parte da população estudada. Os parâmetros avaliados que tinham alguma alteração foram Colesterol (75,7\%) Total e LDL-C (65\%). Fato divergente é observado em outro estudo, onde as alterações foram nos TG (50\%) e no HDL-C (66,6\%) (22).

Dados recentes mostraram que cerca de $17 \%$ dos americanos apresentam níveis de colesterol igual ou acima de $240 \mathrm{mg} / \mathrm{dL}$ e atribuem à hipercolesterolemia o maior fator de risco para o desenvolvimento das doenças cardíacas ${ }^{(23)}$.

Apesar disso, deve-se ter cautela na interpretação desses resultados quando confrontados às prevalências de sujeitos com valores indesejáveis para o perfil lipídico de outras regiões do Brasil, uma vez que diferentes critérios utilizados para classificação das dislipidemias dificultam sua comparação(21).

Com relação à história familiar de doenças, 15,9\% das mães e 13,9\% dos pais tinham hipertensão arterial. Fato também observado em outro estudo, onde $10,2 \%$ das mães e $20,3 \%$ dos pais tinham hipertensão arterial ${ }^{(24)}$.

Um estudo realizado na Finlândia confirmou a presença da agregação familiar na etiopatogenia da hipertensão arterial. Outro estudo verificou que quando o pai ou a mãe tinham a pressão sistólica acima do percentil 97, a pressão sistólica dos filhos era aumentada em 2,7 mm $\mathrm{mg}$ aos 45 anos de idade; quando ambos, pai e mãe, tinham pressão sistólica aumentada, o incremento era de 8,5 mmHg ${ }^{(25,26)}$.

Apesar dos elevados investimentos para o controle das afecções cardiovasculares, as taxas de morbimortalidade têm sofrido poucas modificações. É necessário que a prevenção das DCV seja iniciada na infância, e a equipe de saúde esteja atenta para identificar e intervir precocemente nos seus fatores de risco $^{(23)}$.

Tais fatores de risco quase sempre estão relacionados a aspectos comportamentais. Mudar isso significa fazer com que as pessoas conheçam a sua real situação de saúde e compreendam a necessidade de modificarem seus hábitos de vida. Isto exige habilidades dos profissionais na inserção 
e utilização dos métodos em educação para a saúde na rotina da assistência prestada aos indivíduos, e também maior atenção dos gestores locais na reorganização dos serviços e na priorização das ações desenvolvidas nas unidades de saúde( ${ }^{(27)}$.

A partir do momento que os fatores de riscos para DCVS de uma determinada população passam a ser conhecidos, a comunidade, os serviços de saúde e as escolas da região atuarão com maior controle sobre as decisões e mudanças necessárias para minimizar os riscos e conter os avanços das doenças e agravos, atuando de forma integrada e participativa.

\section{CONCLUSÃo}

De acordo com os resultados evidenciados no presente estudo, podemos observar que os principais fatores de risco para DCV foram: excesso de peso, pressão arterial alterada, colesterol total e LDL-C elevados e HDL-C baixo. Informações dessa natureza se constituem em ferramentas de inquestionável importância para as ações de saúde.

A fase da adolescência é importante devido às mudanças físicas e psicossociais que ocorrem de forma acelerada, facilitando o desenvolvimento de fatores de risco, e por ser uma fase da vida particularmente relevante na aquisição de comportamentos, muitos dos quais permanecem inalterados ao longo da vida.

Com base nesses achados, torna-se obrigatória a formulação de estratégias de prevenção para os adolescentes visando à promoção de estilos de vida saudáveis como dietas com baixa densidade energética, incremento na atividade física e mudanças de comportamento quanto ao controle do peso corporal. Tais estratégias são melhor fundamentadas quando se reconhece a magnitude do problema e apresentam maior eficácia em crianças e adolescentes.

Nesta perspectiva, faz-se necessário que novas pesquisas sejam desenvolvidas com vistas ao melhor delineamento de estratégias de impacto eficazes, seguras e capazes de promover a modificação desse quadro epidemiológico desfavorável, onde as doenças crônicas, principalmente as do aparelho circulatório, passam a ter início cada vez mais cedo.

\section{REFERÊNCIAS}

1. Schmidt MI, Ducan BB, Silva GA, Menezes AM, Monteiro CA, Barreto SM, et al. Doenças crônicas não transmissíveis no Brasil: carga e desafios atuais. The Lancet. 2011. Série Saúde no Brasil; 4.

2. Yokota RTC, Iser BPM, Andrade RLM, dos Santos J, Meiners MMMA, de Assis DM, et al. Vigilância de fatores de risco e proteção para doenças e agravos não transmissíveis em município de pequeno porte, Brasil, 2010. Epidemiol. Serv. Saúde. 2012; 21(1): 55-68.

3. Ministério da Saúde (BR). Secretaria de Vigilância em Saúde. Departamento de Análise de Situação de Saúde. Plano de ações estratégicas para o enfrentamento das doenças crônicas não transmissíveis (DCNT) no Brasil 2011-2022. Brasília: Ministério da Saúde; 2011.

4. Correia BR, Cavalcante E, dos Santos E. A prevalência de fatores de risco para doenças cardiovasculares em estudantes universitários. Rev Bras Clin Med. 2010; 8: 25-9.

5. Canovas CD, Guedes DP. Impacto de diferentes intensidades de caminhada em fatores de risco cardiovasculares em mulheres sedentárias. SaudPesq. 2012; 5(1): 217-24.

6. Bozza R, de Campos W, Stabelini Neto A, da Silva MP, Ulbrich AZ, Mascarenhas LPG, et al. Associação do gasto energético diário com fatores de risco para doença cardiovascular aterosclerótica em adolescentes. R. bras. Ci. e Mov. 2012; 20(4): 69-76.

7. Costa JV, da Silva ARV, de Moura IH, de Carvalho RBN, Bernardes LE, de Almeida PC. Análise de fatores de risco para hipertensão arterial em adolescentes escolares. Rev. Latino-Am. Enfermagem. 2012; 20(2): 289-95. 
8. Luiz RR, Magnanini MMF. O tamanho da amostra em investigações epidemiológicas. In: Medronho A, Bloch KV. Organizadores. Epidemiologia. São Paulo: Atheneu; 2008. p. 295-307.

9. da Silva MAM, Rivera IR, Ferraz MRMT, Pinheiro AJT, Alves SWS, Moura AA, et al. Prevalência de fatores de risco cardiovascular em crianças e adolescentes da rede de ensino da cidade de Maceió. Arq. Bras. Cardiol. 2005; 84(5): 387-92.

10. Molina MCB, de Faria CP, Montero MP, Cade NV, Mill JG. Fatores de risco cardiovascular em crianças de 7 a 10 anos de área urbana, Vitória, Espírito Santo, Brasil. Cad. Saúde Pública. 2010; 26(5): 909-17.

11. Sociedade Brasileira de Cardiologia. Sociedade Brasileira de Hipertensão. VI diretrizes brasileiras de hipertensão. Rev Bras Hipertens. 2010; 17(1): 11-7.

12. National high blood pressure education program working group on high blood pressure in children and adolescents. The fourth report on the diagnosis, evaluation, and treatment of high blood pressure in children and adolescents. Pediatrics. 2004; 114(Suppl 2): 555-76.

13. Sociedade Brasileira de Cardiologia. Sociedade Brasileira de Hipertensão. Sociedade Brasileira de Nefrologia. V Diretrizes Brasileiras de Hipertensão. Arq Bras Cardiol. 2006;(86):1-48.

14. Sociedade Brasileira de cardiologia. I Diretriz de prevenção da aterosclerose na infância e na adolescência. Arq. Bras de Cardiol. 2005; 85(Suppl 6): 3-36.

15. Sociedade Brasileira de Diabetes. Diretrizes da sociedade brasileira de diabetes: 2012-2013. São Paulo: AC Farmacêutica; 2013.

16. Ministério da Saúde (BR). Conselho Nacional de Saúde. Diretrizes e normas regulamentadoras de pesquisa envolvendo seres humanos. Resolução n. 466, de 12 de dezembro de 2012. Brasília; 2012.

17. da Silva PCV, de Araújo MFM, de Almeida LS, de Vasconcelos HCA, de Freitas RWJF, Damasceno MMC, et al. Pressão arterial de adolescentes de escolas particulares de Fortaleza-CE. Acta Paul Enferm. 2010; 23(4): 512-8.

18. Farias Júnior JC, Mendes JKF, Barbosa DBM, Lopes AS. Fatores de risco cardiovascular em adolescentes: prevalência e associação com fatores sociodemográficos. Rev. bras. epidemiol. 2011; 14(1): 50-62.

19. Beck CC, Lopes AS, Giuliano ICB, Borgatto AF. Fatores de risco cardiovascular em adolescentes de município do Sul do Brasil: prevalência e associações com variáveis sociodemográficas. Rev. bras. epidemiol. 2011; 14(1): 36-49.

20. de Souza MGB, Rivera IR, da Silva MAM, Carvalho ACC. Relação da obesidade com a pressão arterial elevada em crianças e adolescentes. Arq. Bras. Cardiol. 2010; 94(6): 714-9.

21. de Campos W, Stabelini Neto A, Bozza R, Ulbrich AZ, Bertin RL, Mascarenhas LPG. et al. Atividade física, consumo de lipídios e fatores de risco para aterosclerose em adolescentes. Arq. Bras. Cardiol. 2010; 94(5): 601-7.

22. Werner MLF, Pone MV, Fonseca VM, Chaves CR. Lipodystrophy syndrome and cardiovascular risk factors in children and adolescents infected with HIVIAIDS receiving highly active antiretroviral therapy. J Pediatr. 2010; 86(1): $27-32$.

23. Ribas SA, da Silva LCS. Dislipidemia em escolares na rede privada de Belém. Arq. Bras. Cardiol. 2009;92(6):44651.

24. Freitas D, Rodrigues CS, Yagui CM, de Carvalho RST, Marchi-Alves LM. Fatores de risco para hipertensão arterial entre estudantes do ensino médio. Acta paul. enferm. 2012; 25(3): 430-4.

25. Moreira AC. Influências familiares de determinantes precoces na ocorrência da obesidade e no risco cardiovascular em crianças [dissertação]. Viçosa (MG): Universidade Federal de Viçosa; 2010.

26. Carnelosso ML, Barbosa MA, Porto CC, Silva SA, Carvalho MM, Oliveira ALI. Prevalência de fatores de risco para doenças cardiovasculares na região leste de Goiânia (GO). Ciênc. saúde coletiva. 2010; 15(Suppl 1): 1073-80. 